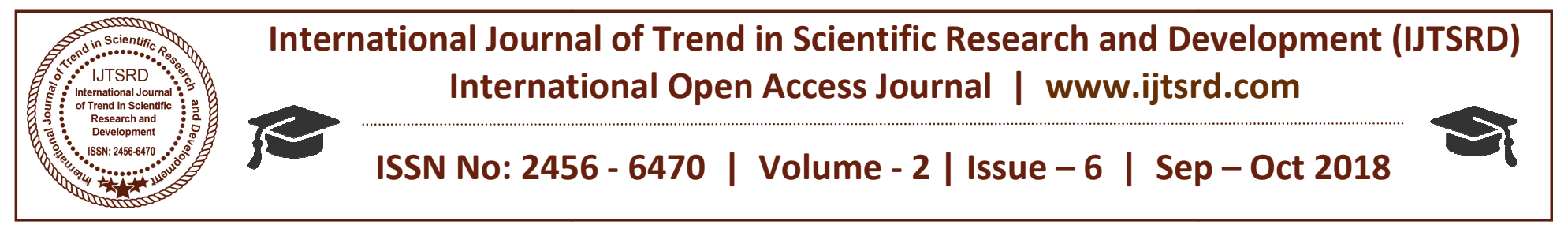

\title{
Observations of can Bus Control System
}

\author{
Aasiya Aslam Tamboli ${ }^{1}$, Prof. Sunil S. Shivdas ${ }^{2}$ \\ ${ }^{1}$ Student, ${ }^{2}$ Professor \\ K.B.P College of Engineering, Shivaji University, Satara, Maharashtra, India
}

\begin{abstract}
One of the important criteria of the modern cars is digital control of the vehicle. Because of the quick development of embedded technology, the high performance embedded processor is inserted into auto industry; it has low cost, high reliability and other features that meet the needs of modern automobile industry. In this project we have introduced an embedded system which is a combination of different CAN bus systems.
\end{abstract}

\section{KEY WORDS: CAN, LM35}

\section{I. $\quad$ MCP 2515}

Microchip technologies MCP 2515 is a controller area network (CAN).It has the ability to transmit and receive both remote and data frames. The MCP2515 has two acceptance masks and six acceptance filters that are used to clean the unwanted messages.

The MCP 2515 has three pins that can be designed as general purpose inputs and two pins that are designed as digital output.The inputs are connected to switch contacts and the outputs to the LED indicators. The MCP 2515 also have internal pull-up resisitors.

Before communicating with the CAN bus, the MCP2515 should be properly configured. The configuration is achieved by loading the control registers with the specific value. During MCP2515 initialization these valus are read via SPI interface. The MCP 2515 interfaces with microcontrollers (MCUs) via SPI i.e. serial peripheral interface.

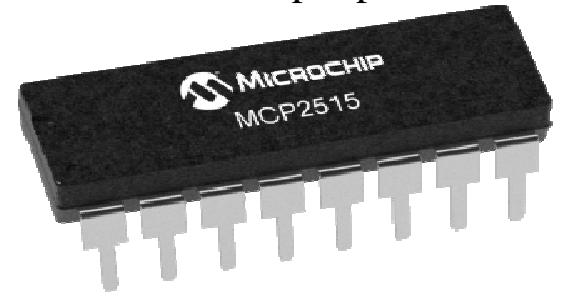

Figure 1: MCP 2515

\section{SPI BUS-}

SPI makes use of different clock,select and data lines to choose the device you wish to talk to.SPI devices communicate in full duplex mode.The SPI bus operates with a single master device and one or more slave devices.

The standard SPI interface has been modifed here which uses commom signal line for both serial in(SI) and serial out(SO) lines. These lines have been isolated from each other via resisitor.This method requires three I/O pins to implement SPI interace.

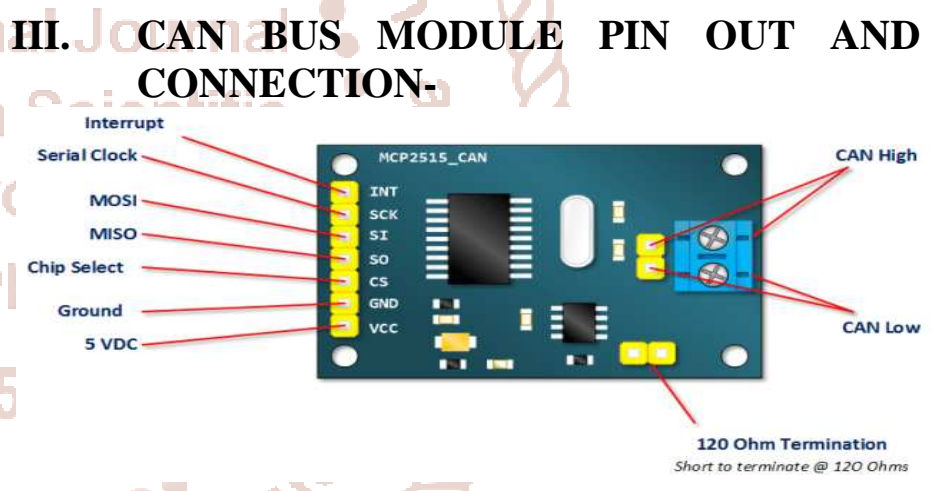

Figure 2: CAN Bus Module

There are variety of CAN applications which include vehicles (via OBDII or J1939) and industrial monitoring and controls. The CAN node is interrupt driven which has the abilty of monitoring five external inputs (i.e. twoanalog and three digital) and automatically creating messages based on their value. This node also controls two digital outputs. The system supports maximum CAN bus speed of $125 \mathrm{kbps}$.

\section{TEMPERATURE SENSOR}

Temperature sensor is basically used to sense the temperature. We have used a Temperature sensor named LM35. This temperature sensor has the ability to sense the temperature of the atmosphere around it 
or the temperature of any machine which is connected to it or even it can give the temperature of the human body. The LM35 sensors have the output voltage which is linearly proportional to the Celsius temperature.

Temperature sensor is an analog sensor and gives the output in analog form. This analog signal is fed to ADC i.e. analog to digital converter, which will convert it into digital form. Once the signal is converted into analog form, the microcontroller can process the digital temperature signal as per the application.

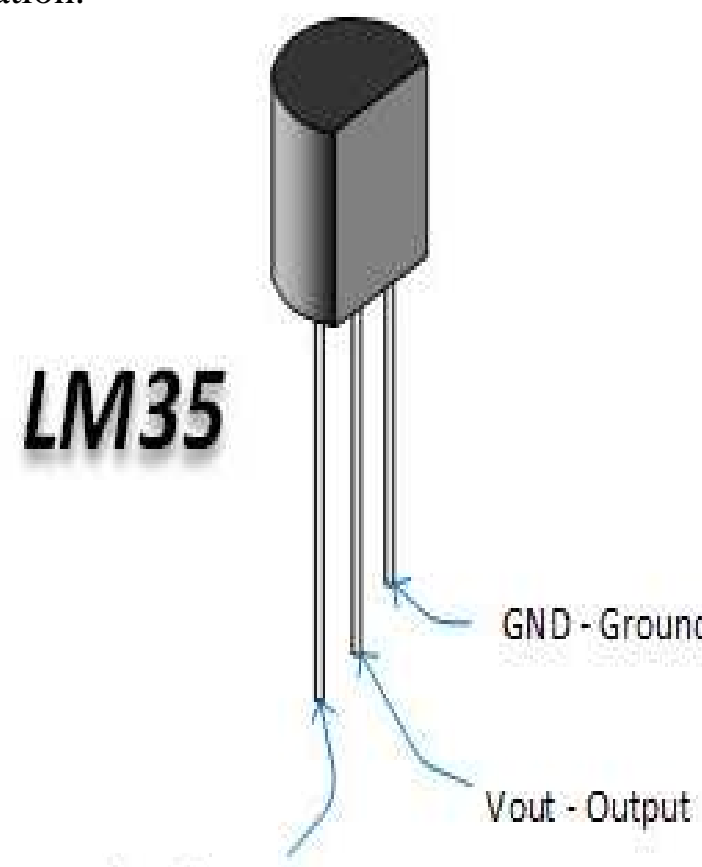

Vs-Supply Voltage

Figure 3: LM35 Temperature sensor

\section{OUTPUT}

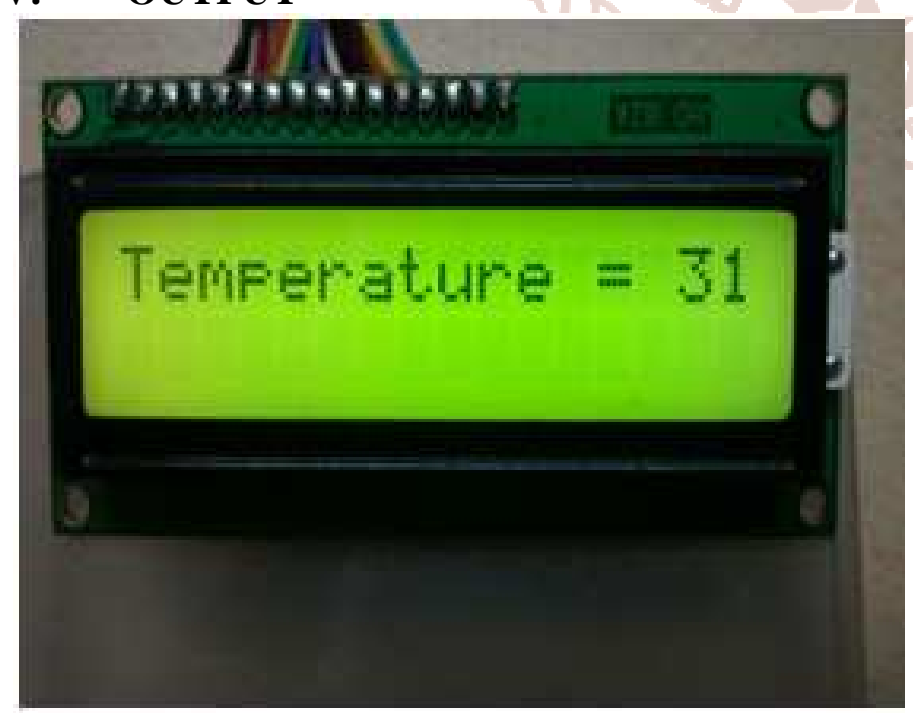

Figure 4: Output on LCD

\section{CONCLUSION}

One of the important criteria of the modern cars is digital control of the vehicle. Because of the quick development of embedded technology, the high performance embedded processor is inserted into auto industry; it has low cost, high reliability and other features that meet the needs of modern automobile industry. In this project we have introduced an embedded system which is a combination of different CAN bus systems.

The proposed high-speed CAN bus system has a practical value and significance, which solves the problem of automotive system applications. In order to achieve full sharing of data between the nodes and to enhance their collaborative work, it has ARM as the main controller which makes full use of the highperformance of ARM. In practical applications, this system promotes efficient data transfer among different node.

Digital control of vehicle is very important criteria. In this project we are introducing a system with a combination of CAN bus system, so that we reduce the wired harness, and maintenance is easy to carry out. Therefore the system becomes safe and reliable.

\section{REFERENCES}

1. Kumar, M., A. Verma, and A. Srividya, ResponseTime Modeling of Controller Area Network (CAN). Distributed Computing and Networking, 2009: p. 163-174.

2. Tindell, K., A. Burns, and A.J. Wellings, Calculating controller area network (CAN) message response times. Control Engineering Practice, 1995. 3(8): p. 1163-1169.

3. Marwedel, P., Embedded System Design: Embedded Systems Foundations of CyberPhysical Systems2010: Springer Verlag.

4. Estrada, B. and P. Mariano, Development of uclinux Platform for Cal Poly Super Project, 2008, California Polytechnic State University.

5. Lu, Z., X. Zhang, and C. Sun. An Embedded System with uClinux based on FPGA. in 2008 Pacific-Asia Workshop on Computational Intelligence and Industrial Application, 2, 691694. 2008. Li, S., et al., Continuous and RealTime Data Acquisition Embedded

6. System for EAST. IEEE Transactions on Nuclear Science,, 2010. 57(2): p. 696-699. 
7. LI, Q. and X. ZHANG, Development of Embedded RTOS uClinux on ARM. Journal of Changchun University of Science and Technology (Natural Science Edition), 2009. 32(2): p. 264267.

8. Li, M., Design of Embedded Remote Temperature Monitoring System Based on Advanced RISC Machine. Electrotechnics Electric, 2009. 6.
9. Zhang, Q.S., et al., Application of Embedded Technology for Induced Polarization Instrument. Advanced Materials Research, 2012. 383: p. 224229.

10. Prodanov, W., M. Valle, and R. Buzas, A controller area network bus transceiver behavioral model for network design and simulation. IEEE Transactions on Industrial Electronics, 2009. 56(9): p. 3762- 3771. 442

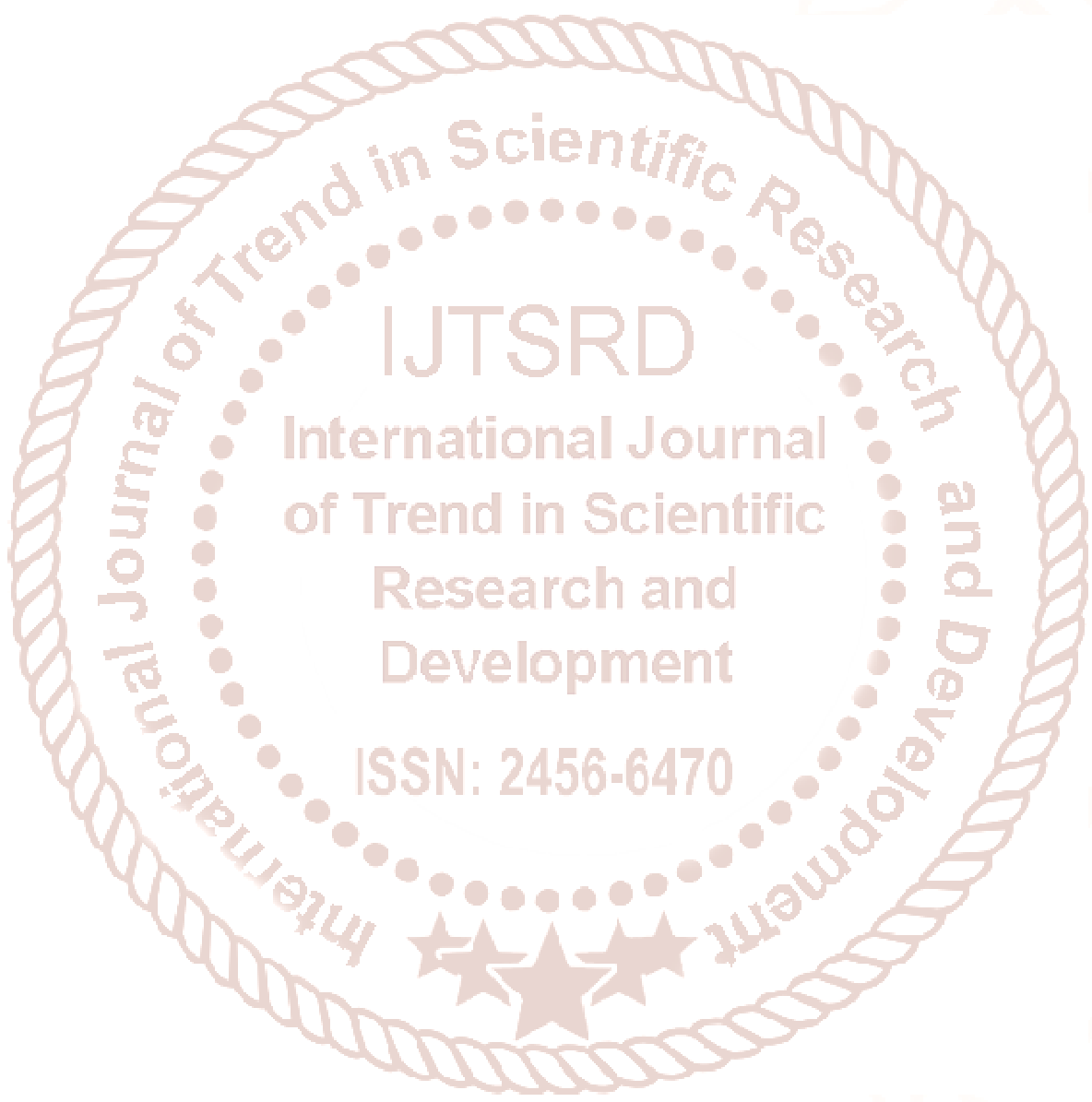

